\title{
The orbit of closure-involution operations: the case of Boolean functions
}

\author{
Jürgen Dassow ${ }^{1}$
}

Received: 31 January 2020 / Accepted: 3 May 2021 / Published online: 15 May 2021

(c) The Author(s) 2021

\begin{abstract}
For a set $A$ of Boolean functions, a closure operator $c$ and an involution $i$, let $\mathcal{N}_{c, i}(A)$ be the number of sets which can be obtained from $A$ by repeated applications of $c$ and $i$. The orbit $\mathcal{O}(c, i)$ is defined as the set of all these numbers. We determine the orbits $\mathcal{O}(S, i)$ where $S$ is the closure defined by superposition and $i$ is the complement or the duality. For the negation non, the orbit $\mathcal{O}(S$, non) is almost determined. Especially, we show that the orbit in all these cases contains at most seven numbers. Moreover, we present some closure operators where the orbit with respect to duality and negation is arbitrarily large.
\end{abstract}

Keywords Kuratowski's closure-complement theorem · Superposition of Boolean functions · Complement and negation and duality of sets of Boolean functions

Mathematics Subject Classification 06D25 -06A15 -08A05

\section{Introduction}

In Kuratowski (1922), proved the following closure-complement theorem: If $(X, \mathcal{T})$ is a topological space and $A \subseteq X$, then at most 14 sets can be obtained from $A$ by repeated applications of the operations topological closure and complement. Furthermore, there is a topological space and a set for which the bound 14 is achieved. More information on Kuratowski-like theorems for topological space can be found in Gardner and Jackson (2008).

Hammer (1960) noticed that such a statement holds in a more general setting; it is not necessary to consider topological spaces and topological closure. The theorem

Dedicated to Prof. Gustav Burosch on the Occasion of his 80th Birthday.

Jürgen Dassow

dassow@iws.cs.uni-magedeburg.de

1 Fakultät für Informatik, Otto-von-Guericke-Universität Magdeburg, PSF 4120, 39106 Magdeburg, Germany 
also holds if - instead of the topological closure - a closure operator on a set $X$ is used.

Most papers related to Kuratowski's closure-complement theorem ask for upper bounds for the number of sets obtainable by repeated applications of a closure operator and complement. However, one can also consider the following more general question: Given a closure operator, determine the set of all numbers $n$ (called the orbit of the closure operator and complement) such that there is a set $A_{n}$ from which we can obtain exactly $n$ sets by repeated applications of the closure operator and complement.

In Brzozowski et al. (2009), this question was firstly investigated by Brzozowski, Grant, and Shallit for the Kleene-closure defined on formal languages and complement. They proved that the orbit of Kleene-closure and complement consists of the numbers $4,6,8,10,12,14$. Moreover, for $n \in\{4,6,8,10,12,14\}$, they gave precise conditions for a language to produce exactly $n$ languages by repeated applications of Kleeneclosure and complement.

For further language theoretic closure operators and involutions (instead of complement) the orbit was studied in Dassow (2019).

In this paper, we continue the determination of the orbit, but we consider the set of Boolean functions. Here a "classical" closure operator is defined by superpositions. The lattice of all closed sets (with respect to superpositions) of Boolean functions was determined in 1921 by Post (see Post 1921, a more complete version is Post 1941, and a modern version is Jablonski et al. 1970). We study the orbit of superpositions and complement, duality, and negation as involution. We prove that the orbit contains three, four, and at most 7 numbers for complement, duality, and negation, respectively. The corresponding Kuratowski numbers are six, four, and seven, respectively.

However, the situation changes completely if we allow other closure operators. We define some special closure operators such that with duality (or negation) the corresponding orbit contains infinity or has $m$ elements where $m$ is an arbitrary natural number with $m \geq 3$.

\section{Definitions and known facts}

Let $X$ be a set. We define the complement $\bar{A}$ of a set $A \subset X$ by $\bar{A}=X \backslash A$.

An operator $c$ is called a closure operator on $X$, if the following three conditions are satisfied:

- For all sets $A \subseteq X, c(A) \subseteq X$.

- For all sets $A \subseteq X, A \subseteq c(A)$.

- For all sets $A \subseteq X$ and $B \subseteq X, A \subseteq B$ implies $c(A) \subseteq c(B)$.

- For all sets $A \subseteq X, c(c(A))=c(A)$, i. e., $c$ is idempotent.

An operator $i$ is called an involution on $X$ if, for any $A \subseteq X$, the relations $i(A) \subseteq X$ and $i(i(A))=A$ hold.

Definition 1 Let $c$ a closure operator on $X$, and $i$ an involution on $X$. Then, for $A \subseteq X$, we define the orbit $\mathcal{O}_{c, i}^{X}(A)$ of $A$ under $c$ and $i$ as the set of all sets which can be obtained 
from $A$ by repeated applications of $c$ and $i$ and set

$$
\mathcal{N}_{c, i}^{X}(A)=\operatorname{card}\left(\mathcal{O}_{c, i}(A)\right)
$$

Moreover, we define the orbit of the pair $(c, i)$ as

$$
\mathcal{O}^{X}(c, i)=\left\{n \mid \mathcal{N}_{c, i}^{X}(A)=n \text { for some } A \subseteq X\right\}
$$

and the Kuratowski number of $(c, i)$ as

$$
\mathcal{K}^{X}(c, i)=\sup \left\{n \mid n \in \mathcal{O}^{X}(c, i)\right\} .
$$

In this terminology, the classical Kuratowski's Theorem is given as follows: If $(X, \mathcal{T})$ is a topological space and $c$ the corresponding topological closure, then $\mathcal{K}^{X}\left(c,{ }^{-}\right)=14$.

By the properties of an closure operator $c$ and an involution $i$, in order to determine the orbit $\mathcal{O}_{c, i}^{X}(A)$ of a set $A$ it is sufficient to determine the sets

$$
A, c(A), i(c(A)), c(i(c(A))), i(c(i(c(A)))), \ldots
$$

and

$$
A, i(A), c(i(A)), i(c(i(A))), c(i(c(i(A)))), \ldots .
$$

Let $P_{2}$ be the set of Boolean functions. In the rest of the paper we restrict to $X=P_{2}$, and for the sake of simplicity we shall omit the upper index $P_{2}$ in the notations given in Definition 1.

We now define some special Boolean functions and sets of Boolean functions, which will be used later. With respect to the notation, we follow (Jablonski et al. 1970).

The constants giving the value 0 or 1 are denoted by $k_{0}$ and $k_{1}$, respectively.

A special unary Boolean function is the negation non defined by $\operatorname{non}(0)=1$ and $\operatorname{non}(1)=0$. We extend the negation to functions by setting $(\operatorname{non}(f))\left(x_{1}, x_{2}, \ldots, x_{n}\right)=\operatorname{non}\left(f\left(x_{1}, x_{2}, \ldots, x_{n}\right)\right)$.

We use the following functions (in some cases we give two notations, and if the functions are associative, we omit some brackets in the sequel):

- $\operatorname{vel}\left(x_{1}, x_{2}\right)=x_{1} \vee x_{2}=0$ if and only if $x_{1}=x_{2}=0$,

- $g^{k}\left(x_{1}, x_{2}, \ldots, x_{k}\right)=x_{1} \vee x_{2} \vee \cdots \vee x_{k}$ for $k \geq 2$,

- et $\left(x_{1}, x_{2}\right)=x_{1} \cdot x_{2}=1$ if and only if $x_{1}=x_{2}=1$,

- $h^{k}\left(x_{1}, x_{2}, \ldots, x_{k}\right)=x_{1} \cdot x_{2} \cdots x_{k}$ for $k \geq 2$,

- $x_{1}+x_{2}=0$ if and only if $x_{1}=x_{2}$,

- $\operatorname{sh}\left(x_{1}, x_{2}\right)=\operatorname{non}\left(\operatorname{vel}\left(x_{1}, x_{2}\right)\right)$ and $\operatorname{sh}^{\prime}\left(x_{1}, x_{2}\right)=\operatorname{non}\left(\operatorname{et}\left(x_{1}, x_{2}\right)\right)$.

Let $C_{2}\left(C_{3}\right)$ be the sets of all functions $f$ such that $f(1,1, \ldots, 1)=1$ $(f(0,0, \ldots, 0)=0$, respectively $)$.

The dual function $d(f)$ of a function $f$ is defined as

$$
(d(f))\left(x_{1}, x_{2}, \ldots, x_{n}\right)=(\operatorname{non}(f))\left(\operatorname{non}\left(x_{1}\right), \operatorname{non}\left(x_{2}\right), \ldots, \operatorname{non}\left(x_{n}\right)\right) .
$$


Moreover, a Boolean function $f$ is called self-dual if and only if $d(f)=f$. Let $D_{3}$ denote the set of all self-dual Boolean functions.

We extend the concept of negation and duality to subsets $A \subseteq P_{2}$ by setting

$$
\operatorname{non}(A)=\{\operatorname{non}(f) \mid f \in A\} \text { and } d(A)=\{d(f) \mid f \in A\} .
$$

Note that these operators non and $d$ are involutions on $P_{2}$.

A function $f$ is called linear if $f\left(x_{1}, x_{2}, \ldots, x_{n}\right)=x_{i_{1}}+x_{i_{2}}+\cdots+x_{i_{r}}$ or $f\left(x_{1}, x_{2}, \ldots, x_{n}\right)=x_{i_{1}}+x_{i_{2}}+\cdots+x_{i_{r}}+1$, where $1 \leq i_{1}<i_{2}<\cdots<i_{r} \leq n$. By $L_{1}$ we denote the family of all linear functions.

By $0<1$, an order is defined on $\{0,1\}$. We say that an $n$-ary function $f$ is monotone if and only if, for all tuples $\left(x_{1}, x_{2}, \ldots, x_{n}\right)$ and $\left(y_{1}, y_{2}, \ldots, y_{n}\right), x_{i} \leq y_{i}$ for $1 \leq i \leq n$ implies $f\left(x_{1}, x_{2}, \ldots, x_{n}\right) \leq f\left(y_{1}, y_{2}, \ldots, y_{n}\right)$.

We now define some operations which lead to the closure operator superposition. For an $n$-ary function $f, n \geq 0$, we set

$$
\begin{aligned}
\left(\zeta_{0}(f)\right)\left(x_{n+1}, x_{1}, x_{2}, \ldots, x_{n}\right) & =f\left(x_{1}, x_{2}, \ldots x_{n}\right) \\
\left(\zeta_{i}(f)\right)\left(x_{1}, x_{2}, \ldots, x_{i}, x_{n+1}, x_{i+1}, \ldots, x_{n}\right) & =f\left(x_{1}, x_{2}, \ldots x_{n}\right), 1 \leq i \leq n .
\end{aligned}
$$

For an $n$-ary function $f, n \geq 2$, and a permutation $\pi$ on $\{1,2, \ldots, n\}$, we define

$$
\begin{aligned}
(\Delta(f))\left(x_{1}, x_{2}, \ldots, x_{n-1}\right) & =f\left(x_{1}, x_{2}, \ldots, x_{n-1}, x_{n-1}\right), \\
(\pi(f))\left(x_{1}, x_{2}, \ldots, x_{n}\right) & =f\left(x_{\pi(1)}, x_{\pi(2)}, \ldots, x_{\pi(n)}\right) .
\end{aligned}
$$

If $f$ is an $n$-ary function and $n \leq 1$, then we set $\Delta(f)=\pi(f)=f$.

For an $n$-ary function $f, n \geq 1$, and an $m$-ary function $g, m \geq 0$, we define the $(n+m-1)$-ary function

$$
(f \circ g)\left(x_{1}, \ldots x_{n-1}, y_{1}, \ldots, y_{m}\right)=f\left(x_{1}, \ldots x_{n-1}, g\left(y_{1}, \ldots, y_{m}\right)\right) .
$$

For a set $A \subseteq P_{2}$, we define [A] as the set of all functions which can be obtained by finitely many iterated application of the operations defined in (1) - (5) to functions from $A$. It is easy to see that the operator $S$ given by $S(A)=[A]$ is a closure operator. Thus, $A$ is called closed if and only if $A=[A]$.

We denote the set of all functions which can be obtained from $f$ by iterated application of (1) and (2) by $\ll f \gg$.

\section{The classical closure operator: superpositions}

In this section we study the orbits of the closure operator given by superpositions and the involutions complement, duality, and negation.

Theorem 1 We have $\mathcal{O}\left(S,^{-}\right)=\{2,4,6\}$. 
Proof Let $A$ be a subset of $P_{2}$. First, we assume that $[A] \neq P_{2}$. Then sh $\notin A$ and sh $\notin[A]\left(\right.$ since $\left.[\{\mathrm{sh}\}]=P_{2}\right)$. Therefore sh $\in \bar{A}$, sh $\in \overline{[A]}$, and $[\bar{A}]=[\overline{[A]}]=P_{2}$. Moreover $\overline{P_{2}}=\emptyset,[\emptyset]=\emptyset$, and $\left[P_{2}\right]=P_{2}$. Consequently, $\mathcal{O}_{S,-}(A)$ contains at most the six sets $A, \bar{A},[A], \overline{[A]}, P_{2}$, and $\emptyset$.

Now let $[A]=P_{2}$. If $[\bar{A}]=P_{2}$, too, then $\mathcal{O}_{S,-}(A)$ contains at most the sets $A, \bar{A}$, $P_{2}$, and $\emptyset$. If $[\bar{A}] \neq P_{2}$, then sh $\left.\in \overline{[\bar{A}}\right]$, which results in $\left.[\overline{[\bar{A}}]\right]=P_{2}$; and consequently at most the six sets $A, \bar{A},[\bar{A}], \overline{[\bar{A}}], P_{2}$, and $\emptyset$ are in $\mathcal{O}_{S,-}(A)$.

Furthermore, if $\mathcal{O}_{S,-}(A)$ contains a set $B$, then it also contains $\bar{B}$. Hence $\mathcal{O}\left(S,{ }^{-}\right)$ contains only even numbers.

Consequently, the only possible numbers which can occur in $\mathcal{O}\left(S,,^{-}\right)$are 2,4, and 6. We now prove that all these numbers are possible.

Let $B_{1}=\{$ vel $\}$. Then $\left[B_{1}\right]=\bigcup_{k \geq 1} \ll g^{k} \gg$ and thus (as shown above) $\left[\overline{B_{1}}\right]=$ $\left[\overline{\left[B_{1}\right]}\right]=P_{2}$. Consequently,

$$
\mathcal{O}_{S,-}\left(B_{1}\right)=\left\{B_{1}, \overline{B_{1}},\left[B_{1}\right], \overline{\left[B_{1}\right]}, P_{2}, \emptyset\right\}
$$

Obviously, the six sets of $\mathcal{O}_{S,-}\left(B_{1}\right)$ are pairwise different. Hence $6 \in \mathcal{O}\left(S,{ }^{-}\right)$.

Let $B_{2}=\{\mathrm{sh}\}$. Then $\operatorname{sh}^{\prime} \in \overline{B_{2}}$ and $\left[B_{2}\right]=\left[\overline{B_{2}}\right]=P_{2}$. Thus

$$
\mathcal{O}_{S,-}\left(B_{2}\right)=\left\{B_{2}, \overline{B_{2}}, P_{2}, \emptyset\right\}
$$

Since these sets are pairwise different, $4 \in \mathcal{O}\left(S,{ }^{-}\right)$.

Let $B_{3}=P_{2}$. Then $\mathcal{O}_{S,-}\left(B_{3}\right)=\left\{P_{2}, \emptyset\right\}$, and consequently $2 \in \mathcal{O}\left(S,^{-}\right)$.

Theorem 2 We have $\mathcal{O}(S, d)=\{1,2,3,4\}$.

Proof Let $A$ be a subset of $P_{2}$. Because $d([A])=[d(A)]$ (Jablonski et al. 1970, Chapter 1, Sect. 6, Folgerung 2), we obtain that $\mathcal{O}_{S, d}(A)$ contains at most the sets $A$, $d(A),[A]$, and $[d(A)]$. Thus the only possible numbers in $\mathcal{O}(S, d)$ are $1,2,3$, and 4 . We now give witnesses for these numbers.

Let $B_{1}=P_{2}$. Then $\mathcal{O}_{S, d}\left(B_{1}\right)=\left\{P_{2}\right\}$ and, consequently, $1 \in \mathcal{O}(S, d)$.

Let $B_{2}=\{g\}$ with $g(x, y, z)=x \cdot \operatorname{non}(y) \vee x \cdot \operatorname{non}(z) \vee \operatorname{non}(y) \cdot z$. Then it is known (see Jablonski et al. 1970) that $g$ is a self-dual function and $[\{g\}]$ is the set $D_{3}$ of all self-dual functions. Thus we obtain $d(\{g\})=\{g\}$ and $d([\{g\}])=[\{g\}]$. This implies $\mathcal{O}_{S, d}\left(B_{2}\right)=\left\{B_{2},\left[B_{2}\right]\right\}$ and $2 \in \mathcal{O}(S, d)$.

Let $B_{3}=\{$ vel, non $\}$. Then we have $d\left(B_{3}\right)=\{$ et, non $\}$ and $[\{$ vel, non $\}]=$ $[\{$ et, non $\}]=P_{2}$ which gives $\mathcal{O}_{S, d}\left(B_{3}\right)=\left\{B_{3}, d\left(B_{3}\right), P_{2}\right\}$. Thus $3 \in \mathcal{O}(S, d)$.

Let $B_{4}=\{$ vel $\}$. Then $d\left(B_{4}\right)=\{$ et $\},\left[B_{4}\right]=\bigcup_{k \geq 1} \ll g^{k} \gg$, and $\left[d\left(B_{4}\right)\right]=$ $\bigcup_{k \geq 1} \ll h^{k} \gg$. Therefore $4 \in \mathcal{O}(S, d)$.

Theorem 3 We have $\{1,2,3,4,5,7\} \subseteq \mathcal{O}(S$, non $) \subseteq\{1,2,3,4,5,6,7\}$.

Proof Let $A$ be a subset of $P_{2}$.

Assume that $[A]=P_{2}$. If $[\operatorname{non}(A)]=P_{2}$ holds, then we have $\mathcal{O}_{S, \text { non }}(A)=$ $\left\{A, \operatorname{non}(A), P_{2}\right\}$ (we do not know whether these sets are pairwise different or some of them are identical). If $[\operatorname{non}(A)] \neq P_{2}$, then $A \subseteq \operatorname{non}([\operatorname{non}(A)])$ and hence 
$[\operatorname{non}([\operatorname{non}(A)])]=P_{2}$. Thus $\mathcal{O}_{S, \operatorname{non}}(A)$ contains at most the sets $A, \operatorname{non}(A),[\operatorname{non}(A)]$, $\operatorname{non}([\operatorname{non}(A)])$, and $P_{2}$.

Now we discuss some cases where $[A] \neq P_{2}$. We start with the cases that $[A]=O_{i}$ for some $i$ with $1 \leq i \leq 9$ where

$$
\begin{array}{lr}
O_{1}=\ll \mathrm{id} \gg & O_{5}=\ll \mathrm{id} \gg \cup \ll k_{1} \gg \\
O_{2}=\ll k_{1} \gg & O_{6}=\ll \mathrm{id} \gg \cup \ll k_{0} \gg \\
O_{3}=\ll k_{0} \gg & O_{7}=\ll k_{1} \gg \cup \ll k_{0} \gg \\
O_{4}=\ll \mathrm{id} \gg \cup \ll \text { non } \gg \quad O_{8}=\ll \mathrm{id} \gg \cup \ll k_{1} \gg \cup \ll k_{0} \gg \\
\\
O_{9}=\ll \mathrm{id} \gg \cup \ll \text { non } \gg \cup \ll k_{1} \gg \cup \ll k_{0} \gg
\end{array}
$$

If $[A]=O_{1}$, then $A \subseteq O_{1}$ and $\operatorname{non}(A) \subseteq \ll$ non $\gg$. Therefore $[\operatorname{non}(A)]=O_{4}$. Furthermore, non $([A])=\operatorname{non}\left(O_{1}\right)=\ll \operatorname{non} \gg$ and $[\operatorname{non}([A])]=O_{4}$. Because $\left[O_{4}\right]=\operatorname{non}\left(O_{4}\right)=O_{4}$ we have that $\mathcal{O}_{S, \text { non }}(A)$ contains at most the sets $A, \operatorname{non}(A)$, $\mathrm{O}_{1}, \ll$ non $\gg$, and $\mathrm{O}_{4}$.

If $[A]=O_{2}$, then $A \subseteq O_{2}$, non $(A) \subseteq O_{3}$, and $[\operatorname{non}(A)]=O_{3}$. Because $\operatorname{non}\left(O_{2}\right)=O_{3}, \operatorname{non}\left(O_{3}\right)=O_{2},\left[O_{2}\right]=O_{2}$, and $\left[O_{3}\right]=O_{3}$, we have that $\mathcal{O}_{S, \text { non }}(A)$ contains at most the sets $A, \operatorname{non}(A), O_{2}$, and $O_{3}$.

If $[A]=O_{3}$, we get analogously that $\mathcal{O}_{S, \text { non }}(A)$ contains at most the sets $A$, non $(A)$, $\mathrm{O}_{2}$, and $\mathrm{O}_{3}$.

If $[A]=O_{4}$, then $A$ contains at least one function $f_{1}$ of $\ll$ non $\gg$. Assume that $A \subseteq \ll$ non $\gg$. Then we get the relations non $(A) \subseteq O_{1}$, $[\operatorname{non}(A)]=O_{1}$, $\operatorname{non}([\operatorname{non}(A)])=\ll \operatorname{non} \gg$, and $[\operatorname{non}([\operatorname{non}(A)])]=O_{4}$. Because non $\left(O_{4}\right)=\left[O_{4}\right]=$ $O_{4}, \mathcal{O}_{S, \text { non }}(A)$ contains at most the sets $A$, non $(A), O_{1}, \ll$ non $\gg$, and $O_{4}$. If we assume that $A$ contains a function of $O_{1}$, too, then non $(A)$ contains a function of $\ll$ non $\gg$ and consequently $[\operatorname{non}(A)]=O_{4}$. Therefore $\mathcal{O}_{S \text {, non }}(A)$ contains at most the sets $A$, non $(A)$, and $O_{4}$.

If $[A]=O_{5}$, then $A$ contains functions $f_{1} \in \ll$ id $\gg$ and $f_{2} \in \ll k_{1} \gg$. Then $\operatorname{non}(A)$ contains a function of $\ll$ non $\gg$ and a function of the set $\ll k_{0} \gg$. This implies $[\operatorname{non}(A)]=O_{9}$. Because we have that non $\left(O_{5}\right)=\ll$ non $\gg \cup \ll k_{0} \gg$, we also obtain $\left[\operatorname{non}\left(O_{5}\right)\right]=O_{9}$. Therefore, $\mathcal{O}_{S, \text { non }}(A)$ contains at most the sets $A, \operatorname{non}(A)$, $\mathrm{O}_{5}$, non $\left(\mathrm{O}_{5}\right)$ and $\mathrm{O}_{9}$.

Analogously, if $[A]=O_{6}$, then $\mathcal{O}_{S, \text { non }}(A)$ contains at most the sets $A$, non $(A)$, $\mathrm{O}_{6}, \operatorname{non}\left(\mathrm{O}_{6}\right)$ and $\mathrm{O}_{9}$.

If $[A]=O_{7}, A$ contains a function of $\ll k_{0} \gg$ and a function of $\ll k_{1} \gg$. This statement holds for non $(A)$, too. Therefore $[\operatorname{non}(A)]=O_{7}$. Because $\left[O_{7}\right]=\operatorname{non}\left(O_{7}\right)=$ $O_{7}, \mathcal{O}_{S, \text { non }}(A)$ contains at most the sets $A$, non $(A)$, and $O_{7}$.

If $[A]=O_{8}, A$ contains functions $f_{1} \in \ll \mathrm{id} \gg$ and $f_{2} \in \ll k_{1} \gg$, and $f_{3} \in \ll k_{0} \gg$. Therefore $\operatorname{non}(A)$ and non([A]) contain non $\left(f_{1}\right) \in \ll$ non $\gg$ and non $\left(f_{2}\right) \in \ll k_{0} \gg$. Hence $[\operatorname{non}(A)]=[\operatorname{non}([A])]=O_{9}$. Thus $\mathcal{O}_{S, \text { non }}(A)$ contains at most the sets $A$, $\operatorname{non}(A), O_{8}$, and $O_{9}$.

If $[A]=O_{9}$, the set $A$ contains a function $f_{1} \in \ll$ non $\gg$ and a function $f_{2} \in \ll k_{i} \gg$ for some $i \in\{0,1\}$. If $A$ contains $f_{3} \in \ll \mathrm{id} \gg$, then we get $[\operatorname{non}(A)]=O_{9}$. Otherwise, $\operatorname{non}(A) \subseteq O_{8}$ and $[\operatorname{non}(A)] \in\left\{O_{5}, O_{6}, O_{8}\right\}$. In all these three cases non $([\operatorname{non}(A)])$ contains a function of $\ll$ non $\gg$ and a function of 
$\ll k_{i} \gg$ for some $i \in\{0,1\}$. Consequently, [non([non $\left.\left.\left.(A)\right]\right)\right]$ equals $O_{9}$. Therefore, $\mathcal{O}_{S, \text { non }}(A)$ contains at most the sets $A, \operatorname{non}(A)$, $[\operatorname{non}(A)], \operatorname{non}([\operatorname{non}(A)])$, and $O_{9}$.

We now discuss the case that $\{$ vel, et $\} \cap[A] \neq \emptyset$. Then we obtain the relation $\left\{\operatorname{sh}, \operatorname{sh}^{\prime}\right\} \cap \operatorname{non}([A]) \neq \varnothing$ which implies $[\operatorname{non}([A])]=P_{2}$. Moreover, $A$ contains a non-self-dual function $f_{1}$ and a non-linear function $f_{2}$ (otherwise $[A]$ would contain only self-dual or only linear functions, which cannot hold by our assumption because vel and et are neither self-dual nor linear. But then non $\left(f_{1}\right)$ is not self-dual and non $\left(f_{2}\right)$ is not linear. Thus [ $A]$ is not contained in $D_{3}$ and not contained in $L_{1}$. Moreover, $A$ has to contain a function $f_{3}$ which depends essentially on at least two variables (otherwise only functions in $\ll$ id $\gg U \ll$ non $\gg \cup \ll k_{0} \gg U \ll k_{1} \gg$ could be generated, which gives $\{$ vel, et $\} \cap[A]=\varnothing$ in contrast to our assumption). Obviously, $\operatorname{non}\left(f_{3}\right)$ also depends on at least two variables. Thus $[\operatorname{non}(A)]$ is not contained in $O_{9}$.

By the Post graph of all closed sets (see Jablonski et al. 1970, page 76), [non $(A)]$ has to contain a function of $\bigcup_{k \geq 1} \ll g^{k} \gg$ or a function of $\bigcup_{k \geq 1} \ll h^{k} \gg$. From this it follows that $\{$ vel, et $\} \cap[\operatorname{non}(\bar{A})]$ is not empty. As above, we get that $[\operatorname{non}([\operatorname{non}(A)])]=$ $P_{2}$. Hence $\mathcal{O}_{S, \text { non }}(A)$ contains at most the sets $A, \operatorname{non}(A),[A], \operatorname{non}([A]),[\operatorname{non}(A)]$, $\operatorname{non}([\operatorname{non}(A)])$, and $P_{2}$.

We now turn to $[A] \in\left\{L_{1}, L_{2}, L_{3}, L_{4}, L_{5}\right\}$ where

$$
L_{2}=L_{1} \cap C_{2}, L_{3}=L_{1} \cap C_{3}, L_{4}=L_{1} \cap C_{2} \cap C_{3}, L_{5}=L_{1} \cap D_{3} .
$$

We note that all functions in $L_{4}$ are self-dual.

Let $[A]=L_{1}$. Then we have that $A \subseteq \operatorname{non}[\operatorname{non}(A)]$. Consequently, we obtain $\left[\operatorname{non}[\operatorname{non}(A)]=L_{1}\right.$. Therefore $\mathcal{O}_{S, \operatorname{non}}(A)$ contains at most the sets $A$, non $(A)$, $[\operatorname{non}(A)] \operatorname{non}([\operatorname{non}(A)])$, and $L_{1}$.

Let $[A]=L_{2}$. Then the set $A$ contains a non-self-dual function $g$. Moreover, id $\in L_{2}$. Hence non $\left(L_{2}\right)$ contains non $(g) \notin D_{3}$ and non $\notin C_{2} \cup C_{3}$. Thus we obtain $\left[\operatorname{non}\left(L_{2}\right)\right]=L_{1}$. Furthermore, $A \subseteq \operatorname{non}([\operatorname{non}(A)])$ which implies the relation $[\operatorname{non}([\operatorname{non}(A)])] \in\left\{L_{2}, L_{1}\right\}$. From the above considerations, we obtain that $\mathcal{O}_{S, \text { non }}(A)$ contains at most the sets $A, \operatorname{non}(A),[\operatorname{non}(A)], \operatorname{non}([\operatorname{non}(A)]), L_{2}, \operatorname{non}\left(L_{2}\right)$, and $L_{1}$.

The case $[A]=L_{3}$ can be handled analogously, and gives also at most seven sets in $\mathcal{O}_{S, \text { non }}(A)$.

Let $[A]=L_{4}$. Since id $\in L_{4}$, we get non $\in \operatorname{non}\left(L_{4}\right)$ and $\left[\operatorname{non}\left(L_{4}\right)\right]=L_{5}$. Moreover, $A \subseteq \operatorname{non}([\operatorname{non}(A)])$ which implies $[\operatorname{non}([\operatorname{non}(A)])] \in\left\{L_{4}, L_{5}\right\}$ and that $\mathcal{O}_{S, \text { non }}(A)$ contains at most the sets $A, \operatorname{non}(A),[\operatorname{non}(A)], \operatorname{non}([\operatorname{non}(A)]), L_{4}, \operatorname{non}\left(L_{4}\right)$, and $L_{5}$.

Let $[A]=L_{5}$. Because $A \subseteq \operatorname{non}([\operatorname{non}(A)])$, we obtain $[\operatorname{non}([\operatorname{non}(A)])]=L_{5}$. Thus $\mathcal{O}_{S, \text { non }}(A)$ contains at most the sets $A, \operatorname{non}(A),[\operatorname{non}(A)], \operatorname{non}([\operatorname{non}(A)])$, and $L_{5}$.

Finally, we discuss the case $[A] \in\left\{D_{1}, D_{2}, D_{3}\right\}$, where $D_{1}$ is the set of all monotone self-dual functions and $D_{2}=D_{3} \cap C_{2} \cap C_{3}$.

Let $[A]=D_{3}$. Because $A \subseteq \operatorname{non}[\operatorname{non}(A)]$, we obtain $\left[\operatorname{non}[\operatorname{non}(A)]=D_{3}\right.$ and that $\mathcal{O}_{S, \text { non }}(A)$ contains at most the sets $A, \operatorname{non}(A),[\operatorname{non}(A)]$, non $([\operatorname{non}(A)])$, and $D_{3}$.

Let $[A]=D_{1}$ or $[A]=D_{2}$. Then id $\in[A]$, and therefore non in non $([A])$, but it is not monotone, not in $C_{2}$ and not in $C_{3}$. Moreover, $A$ and thus non $(A)$ contain 
a non-linear function each. Moreover, for any function $f \in C_{2} \cap C_{3}$, we have that $\operatorname{non}(f) \notin C_{2} \cup C_{3}$ and non $(f)$ is not monotone. Since $D_{2} \subset D_{1} \subset C_{2} \cap C_{3}$, again, by the Post graph of all closed sets, we get $[\operatorname{non}(A)]=D_{3}$. Moreover, $[\operatorname{non}([A])]=D_{3}$ now follows immediately. Hence $\mathcal{O}_{S, \text { non }}(A)$ contains at most the sets $A$, non $(A),[A]$, non ([A]), and $D_{3}$.

By Jablonski et al. (1970), we have above covered all possible cases.

Summarizing, in all cases $\mathcal{O}_{S \text {, non }}(A)$ contains at most seven sets. We now show that the numbers $1,2,3,4,5$, and 7 are possible as the cardinality of some orbit $\mathcal{O}_{S \text {, non }}(A)$.

For $P_{2}$, obviously, we get $\mathcal{O}_{S, \text { non }}\left(P_{2}\right)=\left\{P_{2}\right\}$. Thus $1 \in \mathcal{O}(S$, non $)$.

For $A_{2}=\{\mathrm{sh}$, vel $\}$, we obtain $\operatorname{non}\left(A_{2}\right)=A_{2}$ and $\left[A_{2}\right]=P_{2}$. Consequently, $\mathcal{O}_{S, \text { non }}\left(A_{2}\right)=\left\{A_{2}, P_{2}\right\}$ and hence $2 \in \mathcal{O}(S$, non $)$.

For $A_{3}=\{$ vel, non $\}$, we have non $\left(A_{3}\right)=\{$ sh, id $\}$. Furthermore, we get $\left[A_{3}\right]=$ $\left[\operatorname{non}\left(A_{3}\right)\right]=P_{2}$, which implies $\mathcal{O}_{S \text {, non }}\left(A_{3}\right)=\left\{A_{3}\right.$, non $\left.\left(A_{3}\right), P_{2}\right\}$ and $3 \in \mathcal{O}(S$, non $)$.

For $A_{4}=\left\{k_{0}\right\}$, we obtain the relations non $\left(A_{4}\right)=\left\{k_{1}\right\},\left[A_{4}\right]=\ll k_{0} \gg$, and $\left[\operatorname{non}\left(A_{4}\right)\right]=\ll k_{1} \gg$. Therefore

$$
\mathcal{O}_{S, \text { non }}\left(A_{4}\right)=\left\{\left\{k_{0}\right\},\left\{k_{1}\right\}, \ll k_{0} \gg, \ll k_{1} \gg\right\}
$$

and $4 \in \mathcal{O}(S$, non $)$ hold.

For $A_{5}=\left\{i d, k_{1}\right\}$, we get the relations non $\left(A_{5}\right)=\left\{\right.$ non, $\left.k_{0}\right\},\left[A_{5}\right]=O_{5}$, $\operatorname{non}\left(O_{5}\right)=\ll \operatorname{non} \gg \cup \ll k_{0} \gg$, and $\left[\operatorname{non}\left(A_{5}\right)\right]=\left[\operatorname{non}\left(O_{5}\right)\right]=O_{9}$. Therefore we have

$$
\mathcal{O}_{S, \text { non }}\left(A_{4}\right)=\left\{\left\{\mathrm{id}, k_{1}\right\},\left\{\text { non, } k_{0}\right\}, O_{5}, \ll \text { non } \gg \cup \ll k_{0} \gg, O_{9}\right\}
$$

and $5 \in \mathcal{O}(S$, non).

Let $A_{7}=\{g\}$ where $g(x, y)=x \vee \operatorname{non}(y)$. We recall that $F_{4}^{\infty}$ (and $F_{8}^{\infty}$ ) is the set of all functions $f$ satisfying that there is an $i$ such that $f^{-1}(0) \subseteq\{0,1\}^{i-1} \times$ $\{0\} \times\{0,1\}^{n-i}$ (and $f^{-1}(1) \subseteq\{0,1\}^{i-1} \times\{1\} \times\{0,1\}^{n-i}$, respectively). By Jablonski et al. (1970), $[\{g\}]=F_{4}^{\infty}$. Let $g^{\prime}=\operatorname{non}(g)$. Then $g^{\prime}(x, y)=\operatorname{non}(x) \wedge y$ and $\left[\left\{g^{\prime}\right\}\right]=F_{8}^{\infty}$ (because the function $g^{\prime \prime}(x, y)=x \wedge \operatorname{non}(y)$, which is a generator of $F_{8}^{\infty}$, is obtained from $g^{\prime}$ by a permutation of variables and vice versa). Obviously, the function $h$ with $h(x, y, z)=x \wedge(y \vee z)$ is in $F_{8}^{\infty}$, but non $(h)$ is neither in $F_{8}^{\infty}$ nor in $F_{4}^{\infty}$. By definition, for any $n$-ary function from the set non $\left(F_{4}^{\infty}\right)$, there is a $j$ such that $f^{-1}(1) \subseteq\{0,1\}^{j-1} \times\{0\} \times\{0,1\}^{n-j}$. Now it is obvious that non $(h)$ is not in $\operatorname{non}\left(F_{4}^{\infty}\right)$. By these facts and the infinity of $F_{8}^{\infty}, \operatorname{non}\left(F_{8}^{\infty}\right)$ differs from $A_{7}, \operatorname{non}\left(A_{7}\right)$, $F_{4}^{\infty}, F_{8}^{\infty}$, and non $\left(F_{4}^{\infty}\right)$. Analogously, we can show that non $\left(F_{4}^{\infty}\right)$ differs from $A_{7}$, $\operatorname{non}\left(A_{7}\right), F_{4}^{\infty}, F_{8}^{\infty}$, and non $\left(F_{8}^{\infty}\right)$. Moreover, vel $\in F_{4}^{\infty}$ and et $\in F_{8}^{\infty}$. Therefore $\operatorname{sh} \in \operatorname{non}\left(F_{4}^{\infty}\right), \operatorname{sh}^{\prime} \in \operatorname{non}\left(F_{8}^{\infty}\right)$, and $\left[\operatorname{non}\left(F_{4}^{\infty}\right)\right]=\left[\operatorname{non}\left(F_{8}^{\infty}\right)\right]=P_{2}$. Therefore we get

$$
\mathcal{O}_{S, \text { non }}\left(A_{7}\right)=\left\{A_{7}, \operatorname{non}\left(A_{7}\right), F_{4}^{\infty}, \operatorname{non}\left(F_{4}^{\infty}\right), F_{8}^{\infty}, \operatorname{non}\left(F_{8}^{\infty}\right), P_{2}\right\}
$$

and $7 \in \mathcal{O}(S$, non $)$.

It remains as an open problem whether $6 \in \mathcal{O}(S$, non $)$. We conjecture that six does not belong to $\mathcal{O}(S$, non). The reason for that is that there are only a few cases where 
six can occur (mostly we got that at most five sets are in the orbit of $A$ ), and for some of them we can show that six is impossible.

Corollary 1 With respect to the Kuratowski number the following relations hold:

$$
\mathcal{K}\left(S,^{-}\right)=6, \mathcal{K}(S, d)=4 \text {, and } \mathcal{K}(S, \text { non })=7
$$

\section{Special closure operators}

In the preceding section, we have studied the orbit of superposition and some involutions. In all cases, the Kuratowski number is smaller than 7 and therefore we only get very small orbits. We shall now prove that this depends on the closure operator superposition. If we consider other closure operators on sets of Boolean functions and the involutions duality or negation, we can obtain arbitrary large Kuratowski numbers and arbitrary large orbits.

Theorem 4 There is a closure operation $c_{1}$ such that $\mathcal{O}\left(c_{1}, d\right)=\{1,2,3, \infty\}$.

Proof We say that $f \in P_{2}$ is a $\beta$-function (or $\gamma$-function), if $f(x, \ldots, x)=1$ $(f(x, \ldots, x)=0$, respectively) for $x \in\{0,1\}$. For $n \geq 0$ and $z \in\{\beta, \gamma\}$, by $U(z, n)$, we denote the set of all $n$-ary $z$-functions. Obviously, $U(\beta, 0)=\left\{k_{1}\right\}$ and $U(\gamma, 0)=\left\{k_{0}\right\}$. Hence, for $\kappa \in\{\beta, \gamma\}$, there is no $A$ such that $\emptyset \subset A \subset U(\kappa, 0)$. Moreover, we set

$$
V(\beta, n)=\bigcup_{i=0}^{n} U(\beta, i) \text { and } V(\gamma, n)=\bigcup_{i=0}^{n} U(\gamma, i)
$$

We note that the dual of a $\beta$-function is a $\gamma$-function, and vice versa. Thus $d(U(\beta, n))=U(\gamma, n)$ and $d(U(\gamma, n))=U(\beta, n)$ for $n \geq 0$.

We define $c_{1}$ by

$$
c_{1}(A)= \begin{cases}\emptyset & \text { for } A=\emptyset \\ V(\beta, n+1) & \text { for } A \subseteq V(\beta, n), A \cap U(\beta, n) \neq \emptyset, n \text { even } \\ V(\beta, n) & \text { for } A \subseteq V(\beta, n), A \cap U(\beta, n) \neq \emptyset, n \text { odd } \\ V(\gamma, n+1) & \text { for } A \subseteq V(\gamma, n), A \cap U(\gamma, n) \neq \emptyset, n \text { odd } \\ V(\gamma, n) & \text { for } A \subseteq V(\gamma, n), A \cap U(\gamma, n) \neq \emptyset, n \text { even } \\ P_{2} & \text { otherwise }\end{cases}
$$

We first prove that $c_{1}$ is a closure operator.

(i) The relations $c_{1}(A) \subseteq P_{2}$ and $A \subseteq c_{1}(A)$ follow from the definition of $c_{1}$.

(ii) $A^{\prime} \subseteq A$ implies $c_{1}\left(A^{\prime}\right) \subseteq c_{1}(A)$. We distinguish some cases:

If $A=\emptyset$, then we also have $A^{\prime}=\emptyset$, and $c_{1}\left(A^{\prime}\right)=c_{1}(A)=\emptyset$ holds.

If $A \subseteq V(\beta, n), A \cap U(\beta, n) \neq \emptyset$, and $n$ is even, then, we obtain by definition of $c_{1}, c_{1}(A)=V(\beta, n+1)$. Since $A^{\prime} \subseteq A$, we get $A^{\prime} \subseteq V(\beta, n)$. Let $r$ be the maximal number such that $A^{\prime} \cap U(\beta, r) \neq \varnothing$. If $r=n$, then we get $c_{1}\left(A^{\prime}\right)=V(\beta, n+1)$, 
too, and therefore $c_{1}\left(A^{\prime}\right)=c_{1}(A)$. If $r \leq n-1$, then $c_{1}\left(A^{\prime}\right) \subseteq V\left(\beta, r^{\prime}\right)$ for some $r^{\prime} \leq n-1$, which implies $c_{1}\left(A^{\prime}\right) \subset c_{1}(A)$.

We can analogously prove that $c_{1}\left(A^{\prime}\right) \subseteq c_{1}(A)$ if

$A \subseteq V(\beta, n), A \cap U(\beta, n) \neq \emptyset$, and $n$ is odd, or

$A \subseteq V(\gamma, n), A \cap U(\beta, n) \neq \emptyset$, and $n$ is even, or

$A \subseteq V(\gamma, n), A \cap U(\beta, n) \neq \emptyset$, and $n$ is odd.

If $A$ is not contained in $V(\beta, n)$ and not contained in $V(\gamma, n)$ for some $n$, then $c_{1}(A)=P_{2}$. Therefore $c_{1}\left(A^{\prime}\right) \subseteq c_{1}(A)$ is obvious.

iii) $c_{1}\left(c_{1}(A)\right)=c_{1}(A)$. Again, we distinguish some cases:

If $A=\emptyset$ then $c_{1}(A)=\emptyset$ and $c_{1}\left(c_{1}(A)\right)=c_{1}(\emptyset)=\emptyset$ and $c_{1}\left(c_{1}(A)\right)=c_{1}(A)$ is true.

If $A \subseteq V(\beta, n), A \cap U(\beta, n) \neq \varnothing$, and $n$ is even, then we obtain the relation $c_{1}(A)=V(\beta, n+1)$. Because $c_{1}(A) \cap U(\beta, n+1) \neq \emptyset$ and $n+1$ is odd, we obtain $c_{1}\left(c_{1}(A)\right)=V(\beta, n+1)$ by definition of $c_{1}$, which proves $c_{1}\left(c_{1}(A)\right)=c_{1}(A)$.

If $A \subseteq V(\beta, n), A \cap U(\beta, n) \neq \emptyset$, and $n$ is odd, then $c_{1}(A)=V(\beta, n)$. Because $n$ is odd, $c_{1}\left(c_{1}(A)\right)=V(\beta, n)$, and hence $c_{1}\left(c_{1}(A)\right)=c_{1}(A)$.

Analogously, for $A \subseteq V(\gamma, n)$, we can prove that $c_{1}\left(c_{1}(A)\right)=c_{1}(A)$.

If $L$ is not contained in $V(\beta, n)$ and not contained in $V(\gamma, n)$ for some $n$, then $c_{1}(A)=P_{2}$. Therefore we have $c_{1}\left(c_{1}(A)\right)=c_{1}\left(P_{2}\right)=P_{2}=c_{1}(A)$.

We now determine the orbits of subsets of $P_{2}$.

If $A=\emptyset$ or $A=P_{2}$, we get $\mathcal{O}_{c_{1}, d}(A)=\{A\}$. Therefore $1 \in \mathcal{O}\left(c_{1}, d\right)$.

If $\emptyset \subset A \subset P_{2}$ and $A$ is not contained in $V(\beta, n)$ and not contained in $V(\gamma, n)$ for some $n$, then $d(A)$ satisfies $\emptyset \subset d(A) \subset P_{2}$ and $d(A)$ is not contained in $V(\beta, n)$ and not contained in $V(\gamma, n)$ for some $n$. Thus we obtain $c_{1}(A)=c_{1}(d(A))=P_{2}$ and $\mathcal{O}_{c_{1}, d}(A)=\left\{A, d(A), P_{2}\right\}$. If $A=d(A)$, e.g. for $A=D_{3}$, then $2 \in \mathcal{O}\left(c_{1}, d\right)$. If $A \neq d(A)$, e.g. for $A=\{$ et $\}$, then $3 \in \mathcal{O}\left(c_{1}, d\right)$.

Let $A \subseteq V(\beta, n), A \cap U(\beta, n) \neq \varnothing$, and $n$ even. Starting with the closure operator $c_{1}$, we obtain the following infinite sequences of sets:

A,

$$
\begin{aligned}
& c_{1}(A)=V(\beta, n+1), \\
& d\left(c_{1}(A)\right)=V(\gamma, n+1), \\
& c_{1}\left(d\left(c_{1}(A)\right)\right)=V(\gamma, n+2), \\
& d\left(c_{1}\left(d\left(c_{1}(A)\right)\right)\right)=V(\beta, n+2), \\
& c_{1}\left(d\left(c_{1}\left(d\left(c_{1}(L)\right)\right)\right)\right)=V(\beta, n+3), \\
& d\left(c_{1}\left(d\left(c_{1}\left(d\left(c_{1}(L)\right)\right)\right)\right)\right)=V(\gamma, n+3), \\
& \ldots
\end{aligned}
$$

which proves that $\mathcal{O}_{c_{1}, d}(A)$ is an infinite set. (For the sake of completeness we mention that the sequence starting with duality gives 


$$
\begin{aligned}
& A, \\
& d(A), \\
& c_{1}(d(A))=V(\gamma, n), \\
& d\left(c_{1}(d(A))\right)=V(\beta, n) \\
& c_{1}\left(d\left(c_{1}(d(A))\right)\right)=V(\beta, n+1)=c_{1}(A), d\left(c_{1}(A)\right), \ldots
\end{aligned}
$$

which is up to first elements the same sequence which was obtained by starting with the closure operator.)

Analogously we can prove that we have infinite orbits for sets $A$ where $A \subseteq V(\beta, i)$, $A \cap U(\beta, n) \neq \varnothing$, and $n$ is odd or $A \subseteq V(\gamma, i)$ for some $n$.

Since we have covered all possible cases, $\mathcal{O}\left(c_{1}, d\right)=\{1,2,3, \infty\}$ follows.

Theorem 5 For any positive integer $n$, there is a closure operation $c_{2}$ such that $\mathcal{O}\left(c_{2}, d\right)=\{1,3\} \cup\{2,4, \ldots, 2 n+2\}$.

Proof Using the notation of the preceding proof, we define $c_{2}$ by

$$
c_{2}(A)= \begin{cases}\emptyset & \text { for } A=\emptyset \\ V(\beta, k+1) & \text { for } A \subseteq V(\beta, k), A \cap U(\beta, k) \neq \emptyset, k \text { even, } k<n, \\ V(\beta, k) & \text { for } A \subseteq V(\beta, k), A \cap U(\beta, k) \neq \emptyset, k \text { even, } k \geq n, \\ V(\beta, k) & \text { for } A \subseteq V(\beta, k), A \cap U(\beta, k) \neq \emptyset, k \text { odd, } \\ V(\gamma, k+1) & \text { for } A \subseteq V(\gamma, k), A \cap U(\gamma, k) \neq \emptyset, k \text { odd, } k<n, \\ V(\gamma, k) & \text { for } A \subseteq V(\gamma, k), A \cap U(\gamma, k) \neq \emptyset, k \text { odd, } k \geq n, \\ V(\gamma, k) & \text { for } A \subseteq V(\gamma, k), A \cap U(\gamma, k) \neq \emptyset, k \text { even, } \\ P_{2} & \text { otherwise }\end{cases}
$$

Analogously to the proof of Theorem 4, we can show that $c_{2}$ is a closure operator.

If $A=\emptyset$ or $A=P_{2}$, we obtain $\mathcal{O}_{c_{2}, d}(A)=\{A\}$ and thus $1 \in \mathcal{O}\left(c_{2}, d\right)$.

If $A$ is not contained in some $V(\beta, k)$ and not contained in some $V(\gamma, k), k \geq 0$, then $d(A)$ is also not contained in some $V(\beta, k)$ and not contained in some $V(\gamma, k)$, and we obtain $\mathcal{O}_{c_{2}, d}(A)=\left\{A, d(A), P_{2}\right\}$ which gives $2,3 \in \mathcal{O}\left(c_{2}, d\right)$ (as in the proof of Theorem 4).

If $A \subset V(\beta, k), A \cap U(\beta, k) \neq \emptyset$, and $0 \leq k<n$, then we obtain

$$
\mathcal{O}_{c_{2}, d}(A)=\{A, d(A)\} \cup\{V(\beta, k) \mid k \leq r \leq n\} \cup\{V(\gamma, k) \mid k \leq r \leq n\} .
$$

We show this fact only for even $k$ and even $n$ (the proof for the other cases can be given analogously). If we start with $c_{2}$, we get the following sets (which are obtained in succession)

$$
\begin{aligned}
& V(\beta, k+1), V(\gamma, k+1), V(\gamma, k+2), V(\beta, k+2), \\
& V(\beta, k+3), V(\gamma, k+3), \ldots, \\
& V(\gamma, n-2), V(\beta, n-2), V(\beta, n-1), V(\gamma, n-1), \\
& V(\gamma, n), V(\beta, n), V(\beta, n), V(\gamma, n), V(\gamma, n), V(\beta, n), \ldots,
\end{aligned}
$$


and if we start with $d$, we get $d(A), V(\gamma, k), V(\beta, k), V(\beta, k+1)$ and continue as above (where we started with $c_{2}$ ). Therefore

$$
\mathcal{N}_{c_{2}, d}(A)=2(n-k+1)+2
$$

If $A=V(\beta, k)$ and $0 \leq k<n$, we obtain $\mathcal{N}_{c_{2}, d}(A)=2(n-k+1)$.

If $A \subseteq V(\gamma, k)$ and $k<n, \mathcal{N}_{c_{2}, d}(A) \in\{2(n-k+1), 2(n-k+1)+2\}$ follows by slight modifications of the above consideration.

If $A \subset V(\beta, k)$ and $A \cap U(\beta, k) \neq \emptyset$ or $A \subset V(\gamma, k)$ and $A \cap U(\gamma, k) \neq \emptyset$ for some $k \geq n$, then we obtain $\mathcal{O}_{c_{2}, d}(A)=\{A, d(A), V(\beta, k), V(\gamma, k)\}$. If $A=V(\beta, k)$ or $A=V(\gamma, k)$ for some $k \geq n$, then $\mathcal{O}_{c_{2}, d}(A)=\{V(\beta, k), V(\gamma, k)\}$ holds.

Summarizing these facts, we obtain $\mathcal{O}\left(c_{2}, d\right)=\{1,3\} \cup\{2,4, \ldots, 2 n+2\}$.

Theorem 6 For any positive integer $n$, there is a closure operation $c_{3}$ such that $\mathcal{O}\left(c_{3}, d\right)=\{1,2,3\} \cup\{5,7, \ldots, 2 n+3\}$.

Proof We define $c_{3}$ as follows:

$$
c_{3}(A)= \begin{cases}\emptyset & \text { for } A=\emptyset \\ V(\beta, k+1) & \text { for } A \subseteq V(\beta, k), A \cap U(\beta, k) \neq \emptyset, k \text { even, } k<n \\ V(\beta, k) & \text { for } A \subseteq V(\beta, k), A \cap U(\beta, k) \neq \emptyset, k \text { odd, } k<n \\ V(\gamma, k+1) & \text { for } A \subseteq V(\gamma, k), A \cap U(\gamma, k) \neq \emptyset, k \text { odd, } k<n \\ V(\gamma, k) & \text { for } A \subseteq V(\gamma, k), A \cap U(\gamma, k) \neq \emptyset, k \text { even, } k<n \\ P_{2} & \text { otherwise }\end{cases}
$$

Now we follow the lines of the preceding proof; the only difference is that, for $A \subseteq V(\beta, n)$ and $A \subseteq V(\gamma, n)$ we additionally get $P_{2}$ in $\mathcal{O}_{c_{3}, d}(A)$.

We mention that statements analogous to the Theorems 4, 5 and 6 also hold for the involution non. We only do the following changes: Instead of $\beta$-functions we take functions from $C_{2} \cap C_{3}$ and instead of $\gamma$-functions we take functions which are not in $C_{2} \cup C_{3}$. Then we have the property that the negation of a function in $C_{2} \cap C_{3}$ is not in $C_{2} \cup C_{3}$ and vice versa.

We note that we cannot obtain arbitrary sets of natural numbers as orbits with respect to some closure operator and duality or negation. This comes from the fact that the following statement was shown in Dassow (2019): Let c be a closure operator on $X$ and $i$ an involution on $X$ such that $A^{\prime} \subseteq A \subseteq X$ implies $i\left(A^{\prime}\right) \subseteq i(A)$. If $\mathcal{K}^{X}(c, i) \geq 6$, then

$$
\left\{2 k+1 \mid 0 \leq k \leq \frac{\mathcal{K}^{X}(c, i)}{4}-1\right\} \subset \mathcal{O}^{X}(c, i)
$$

or

$$
\left\{2 k \mid 1 \leq k \leq \frac{\mathcal{K}^{X}(c, i)}{4}-1\right\} \subset \mathcal{O}^{X}(c, i) .
$$

Consequently, because $d$ and non satisfy the suppositions for $i$, certain "small" numbers have to be in $\mathcal{O}(c, d)$ and $\mathcal{O}(c$, non $)$. 


\section{Conclusion}

In this paper we started the investigation of the Kuratowski number $\mathcal{K}(c, i)$ and the orbit $\mathcal{O}(c, i)$ for some closure operators $c$ on the set $P_{2}$ of all Boolean functions and some involutions $i$ on $P_{2}$.

Especially we have determined the orbits $\mathcal{O}\left(S,{ }^{-}\right)$and $\mathcal{O}(S, d)$ where $S$ is the operator given by the closure defined by superposition and the involution complement or duality, respectively. For superposition and the involution negation, we have proved that $\mathcal{K}(S$, non $)=7$ and with respect to the orbit $\mathcal{O}(S$, non $)$, we have only left open whether or not $6 \in \mathcal{O}(S$, non $)$.

Furthermore, we have presented some closure operators $c$ where the orbits $\mathcal{O}(c, d)$ and $\mathcal{O}(c$, non) are arbitrarily large. It remains as an open problem to characterize the sets of natural numbers which can occur as orbits $\mathcal{O}(c, i)$.

Furthermore, we mention that it remains to study the Kuratowski number and the orbits if the basic set is the set $P_{k}$ of all functions which map $\{0,1,2, \ldots, k-1\}^{n}$ into $\{0,1,2, \ldots, k-1\}$ for some $n$, i.e., of all functions of $k$-valued logic. By our results and their proofs (the functions

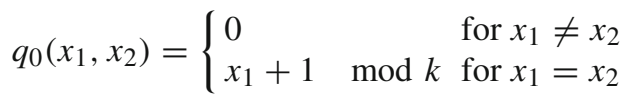

and

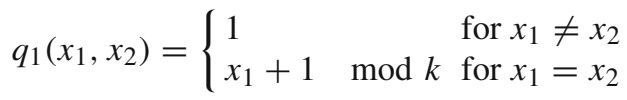

serve as sh and $\mathrm{sh}^{\prime}$, respectively), we obtain

$$
\mathcal{O}^{P_{k}}\left(S,{ }^{-}\right)=\{2,4,6\} \text { and } \mathcal{K}\left(S,{ }^{-}\right)=6 .
$$

Let $f$ be a unary function of $P_{k}$ such that $f(f(x))=x$ for all values $x$ in $\{0,1,2, \ldots, k-1\}$. Then we can define an $f$-duality $d_{f}$ by

$$
\left(d_{f}(g)\right)\left(x_{1}, x_{2}, \ldots, x_{n}\right)=f\left(g\left(f\left(x_{1}, f\left(x_{2}\right), \ldots, f\left(x_{n}\right)\right)\right)\right.
$$

and a "negation" $\left(\operatorname{non}_{f}(g)\right)\left(x_{1}, \ldots, x_{n}\right)=f\left(g\left(x_{1}, \ldots, x_{n}\right)\right)$. We have no results concerning these involutions.

Funding Open Access funding enabled and organized by Projekt DEAL.

Open Access This article is licensed under a Creative Commons Attribution 4.0 International License, which permits use, sharing, adaptation, distribution and reproduction in any medium or format, as long as you give appropriate credit to the original author(s) and the source, provide a link to the Creative Commons licence, and indicate if changes were made. The images or other third party material in this article are included in the article's Creative Commons licence, unless indicated otherwise in a credit line to the material. If material is not included in the article's Creative Commons licence and your intended use is not permitted by statutory regulation or exceeds the permitted use, you will need to obtain permission directly from the copyright holder. To view a copy of this licence, visit http://creativecommons.org/licenses/by/4.0/. 


\section{References}

Brzozowski, J., Grant, E., Shallit, J.: Closures in formal languages and Kuratowski's theorem. In: Diekert, V., Nowotka, D.(eds.), Development of Languages, LNCS 5583, Springer-Verlag, pp. 125-144 (2009)

Charlier, É., Domaratzki, M., Harju, T., Shallit, J.: Finite orbits of language operations. In: Dediu, A.-H., Martin-Vide, C., Inemaga, Sh. (eds.): Languages and Automata, Theory and Application, LNCS 6638, Springer-Verlag, pp. 204-215 (2011)

Dassow, J.: On the orbit of closure-involution operations: the case of formal languages. Theoret. Comput. Sci. 777, 192-203 (2019)

Gardner, B.J., Jackson, M.: The Kuratowski closure-complement theorem. N. Zeal. J. Math. 38, 9-44 (2008) Hammer, P.C.: Kuratowski's closure theorem. Nieuw Archief v. Wiskunde 7, 74-80 (1960)

Jablonski, S.W., Gawrilow, G.P., Kudrjawzew, W.B.: Boolesche Funktionen und Postsche Klassen. Akademie-Verlag, Berlin (1970)

Kuratowski, K.: Sur l'opération $\bar{A}$ de l'analysis situs. Fund. Math. 3, 182-199 (1922)

Post, E.L.: Introduction to a general theory of elementary propositions. Am. J. Math. 43, 163-185 (1921)

Post, E.L.: The two-valued iterative systems of mathematical logic. Princeton University Press, Annals of Mathematics Studies (1941)

Publisher's Note Springer Nature remains neutral with regard to jurisdictional claims in published maps and institutional affiliations. 